Pacific Journal of Mathematics

DESCRIPTION OF HSP-LIKE CLASSES, AND 


\title{
A DESCRIPTION OF HSP-LIKE CLASSES, AND APPLICATIONS
}

\author{
ANThony W. Hager
}

\begin{abstract}
First, in a concrete category, an $\mathrm{HS}^{\prime} \mathrm{P}$-class (of objects) is one closed under P: products, $S^{\prime}$ : some kind of subobjects, $H$ : surjective images. Next, given a class $E$ of morphisms, the object class of "injectives for $E$ " is defined: $A \in \operatorname{inj} E$ means $\forall e \in E, \forall \varphi \in$ $\operatorname{Hom}(\operatorname{domain}(e), A), \exists \bar{\varphi} \in \operatorname{Hom}(\operatorname{codomain}(e), A)$ with $\bar{\varphi} e=\varphi$. Then, the "description" of the title is, in a concrete category with enough free objects, and well-behaved in other ways: the $\mathrm{HS}^{\prime} \mathrm{P}$-classes are exactly the classes of the form inj $E$, for just those $E$ which have domain $(e)$ free for each $e \in E$ (with the meaning of $S^{\prime}$ and the nature of the maps in $E$ depending on each other). This includes a version of Birkhoff's Variety Theorem, but more to the present point, is interpreted easily in various specific settings from topology, algebra, and abstract analysis to provide quite concrete descriptions of HSP-like classes.
\end{abstract}

1. S'P-classes. In this preliminary section, we assemble definitions and facts needed for the main result (2.2 below). A detailed account of most of this appears in [HS, Chapter X] (though the Remark below notes a small caveat).

1.1. Definitions. Let $\mathscr{C}$ be a category. A "class of objects" in $\mathscr{C}$, or a "subcategory of $\mathscr{C}$ " always will be assumed isomorphism-closed, and subcategories will be assumed full.

A. The subcategory $\mathscr{R}$ is reflective in $\mathscr{C}$ if, for each object $C \in \mathscr{C}$ there is an object $r C \in \mathscr{R}$ (the reflection) and a map $C \stackrel{r_{c}}{\rightarrow} r C$ (the reflection map) such that, whenever $R \in \mathscr{R}$ and $\varphi \in \operatorname{Hom}(C, R)$ there is unique $\bar{\varphi} \in \operatorname{Hom}(r C, R)$ with $\bar{\varphi} r_{c}=\varphi$.

The class $\mathscr{A}$ of objects is a $P$-class if whenever $\left\{A_{l}\right\}$ is a subject of $\mathscr{A}$, then the $\mathscr{C}$-product $\pi_{i} A_{i}$ exists and lies in $\mathscr{A}$.

B. Let $\mathscr{E} \subseteq$ epics (in $\mathscr{C}$ ) be closed under composition and contain all isomorphisms.

$\mathscr{R}$ is $\mathscr{E}$-reflective if $\mathscr{R}$ is reflective with each $r_{c} \in \mathscr{E}$.

Define $\mathscr{M}(\mathscr{E})=\{m \mid m=g e, e \in \mathscr{E} \Rightarrow e$ isomorphism $\}$. If $B \stackrel{m}{\rightarrow} A \in$ $\mathscr{M}(\mathscr{E})$, we call $B$ an $\mathscr{M}(\mathscr{E})$-subobject of $A$. 
Class $\mathscr{A}$ of objects is an $S_{\mathscr{E}}$-class if $A \in \mathscr{A}$ and $B \stackrel{m}{\rightarrow} A \in \mathscr{M}(\mathscr{E}) \Rightarrow$ $B \in \mathscr{A}$.

We call $\mathscr{C}$ an $\mathscr{E}$-category if $(a) \mathscr{M}(\mathscr{E})$ is closed under composition, and each morphism $f$ has a factorization $f=m e$, with $e \in \mathscr{E}$ and $m \in \mathscr{M}(\mathscr{E})$, which is unique up to isomorphism, and (b) $\mathscr{C}$ is $\mathscr{E}$-cowellpowered, i.e., each object has a "sufficient set" of $\mathscr{E}$-maps out of it.

REMARK. In the above, one may think of $\mathscr{E}=$ surjections and $\mathscr{M}(\mathscr{E})$ = injections, whence " $\mathscr{M}(\mathscr{E})$-subobject" is just "subobject" (in many familiar categories, at least), or of $\mathscr{E}=$ epics, whence the maps in $\mathscr{M}(\mathscr{E})$ are called extremal monic.

However, while our applications in $\S 3,4$ are of that sort, we should note (the referee points out) that, in general, $\mathscr{M}(\mathscr{E})$ need not consist of monics. In fact, $33 \mathrm{H}$ of [HS] is an example, relevant to our 4.1 below, which see. (Condition (a) in the definition of $\mathscr{E}$-category says that $(\mathscr{E}, \mathscr{M}(\mathscr{E}))$ is a "right bicategory structure" in the sense of $\left[\mathbf{K}_{\mathbf{2}}\right]$. This differs from the "( $\mathscr{E}, \mathscr{M}(\mathscr{E}))$-category" of [HS] exactly in that our $\mathscr{M}(\mathscr{E})$ need not consist of monics.)

Now recall the definition of inj $E$ in the introduction.

1.2. THEOREM. Let $\mathscr{C}$ be an $\mathscr{E}$-category with products.

(a) (Freyd, Isbell, Kennison) The subcategory $\mathscr{R}$ is $\mathscr{E}$-reflective iff the object class of $\mathscr{R}$ is an $S_{\mathscr{E}} P$-class.

(b) (Isbell, Kennison) The class of objects $\mathscr{R}$ is an $S_{\mathscr{E}} P$-class iff $R=\operatorname{inj} E$ for some $E \subseteq \mathscr{E}$.

1.2(a) is the cornerstone of the theory of reflections. Exactly this is stated and proved by Kennison in [ $\mathbf{K}_{2}$, p. 356]. Kennison attributes the result to Freyd and Isbell, referring to $\left[\mathbf{I}_{2}\right]$. In $\left[\mathbf{I}_{2}\right.$, p. 1276], Isbell states and proves the above assuming $\mathscr{M}(\mathscr{E}) \subseteq$ monics, says "... except for quibbles, it is a result of Freyd.", and refers to Freyd's thesis. One may also see [HS], which has $\mathscr{M}(\mathscr{E}) \subseteq$ monics; that makes little difference to the proof.

Our Theorem here (the introduction and 2.2 below) is clearly a refinement of 1.2(b), regarding which: $1.2(\mathrm{~b})$ is implicit in $\left[\mathbf{I}_{\mathbf{2}}\right]$ and $\left[\mathbf{K}_{\mathbf{1}}\right]$. In [I $\mathbf{I}_{1}$, one may begin looking at p. 644: 3.24 must use a version. In [ $\mathbf{K}_{\mathbf{1}}$, see p. 406, "Proof of Theorem 1.2"; here, however, $\mathscr{E}=$ all epics. Doubtless, the idea of using injectivity classes has appeared in many other places.

One proves 1.2 (b) as follows: If $\mathscr{R}$ is $S_{\mathscr{E}} P$, then $\mathscr{R}$ is $\mathscr{E}$-reflective by 1.2(a). Then, let $E=\left\{r_{c} \mid C \in \mathscr{E}\right\}$ (i.e., all reflection maps for $\mathscr{R}$ ). It is 
easy to prove that $\mathscr{R}=\operatorname{inj} E$. Conversely, if $\mathscr{R}=\operatorname{inj} E:$ It is routine and standard to verify that $\mathscr{R}$ is a $P$-class. It is routine too that $\mathscr{R}$ is an $S_{\mathscr{E}}$-class, using, though, the important "diagonalization" lemma ([K $\left.\mathbf{K}_{\mathbf{2}}\right]$, 1.1(6)): If $g e=m f$, with $e \in \mathscr{E}$ and $m \in \mathscr{M}(\mathscr{E})$, then there is $k$ with $k e=f$ and $m k=g$. (The crucial role of diagonalization is made clear in [HS].)

2. $\mathrm{HS}_{\mathscr{E}} \mathrm{P}$-classes. The following is the setting for our description, 2.2 below.

2.1. Let $\mathscr{C}$ be a category, with a functor $\mathscr{C} \stackrel{\mu}{\rightarrow} \mathscr{G}$ to another category $\mathscr{G}$. (Usually, $\mathscr{G}$ will be the category of sets and functions, with $\mu$ an obvious forgetful functor.)

(1) A morphism $A \stackrel{\sigma}{\rightarrow} Q$ of $\mathscr{C}$ will be called a surjection (in $\mathscr{C}$ ) if $\mu \sigma$ is a retraction in $G$, i.e., if there is $\mu A \stackrel{\chi}{\leftarrow} \mu Q$ with $(\mu \sigma) \chi=\mathrm{id}_{\mu Q}$. (In case $\mathscr{G}$ is sets and functions, we shall, of course, want "onto homomorphisms" to be surjections: we assume the Axiom of Choice.)

A class $\mathscr{A}$ of objects will be called an $H$-class if $A \in \mathscr{A}$ and $A \stackrel{\mu}{\rightarrow} Q$ a surjection imply $Q \in \mathscr{A}$.

(2) We shall suppose that $\mu$ has a left adjoint, i.e., over each $\mathscr{G}_{\text {-object }}$ there is a $\mu$-free object, i.e.:

Given $G \in \mathscr{G}$, there is $F(G) \in \mathscr{C}$ and a $\mathscr{G}$-map $G \stackrel{\iota_{G}}{\rightarrow} \mu F(G)$ such that whenever $C \in \mathscr{C}$ and $G \stackrel{g}{\rightarrow} \mu C$ is a $\mathscr{G}$-map, there is a unique $\mathscr{C}$-map $F(G) \stackrel{\bar{g}}{\rightarrow} C$ with $(\mu \bar{g})_{\iota_{G}}=g$.

The objects of the form $F(G)$ are called free.

2.2. THEOREM. Let $\mathscr{C}$ be an $\mathscr{E}$-category with products, which satisfies 2.1 , and let $\mathscr{R}$ be a class of objects. Then $\mathscr{R}$ is an $H S_{\mathscr{E}} P$-class iff $\mathscr{R}=\operatorname{inj} E$ for some $E \subseteq \mathscr{E}$ with $\operatorname{domain}(e)$ free for each $e \in E$.

2.3. REMARKS. In 2.2, note that: $\mu$ appears in the definition of $H$, and in the definition of free; $\mathscr{E}$ appears in $S_{\mathscr{E}}$ and in the condition " $E \subseteq \mathscr{E}$ "; $\mu$ and $\mathscr{E}$ have almost nothing to do with each other. (One may note that since retractions are always epic, if $\mu$ is faithful, then surjections are epic, but not necessarily in $\mathscr{E}$.)

Thus, if one wants to study epireflections in a particular $\mathscr{C}$ via 2.2, there are two degrees of freedom, choice of $\mathscr{E}$ and choice of $\mu$. These choices are illustrated in 4.1 and 4.5 , below. 
(I don't have an example where it is definitely necessary to use some $\stackrel{\mu}{\rightarrow}$ other than a relatively obvious underlying set or unit ball functor. It seems to me that there surely are such examples. In any event, there is no technical complication at all associated with our apparent additional generality.)

Proof of 2.2. We show that any $\mathrm{HS}_{\mathscr{E}} \mathrm{P}$-class takes the indicated form.

(1) Lemma. (Slight variant of 31.9 , [HS]). In $\mathscr{C}$, each object is the surjective image of a free object.

Proof. Given $A$, let $F(\mu A) \stackrel{f}{\rightarrow} A$ be the unique map for which $(\mu f) \iota_{\mu A}=\mathrm{id}_{\mu A}($ per 2.1(2)). Then immediately, $\mu f$ is a retraction in $G$, hence $f$ is a surjection.

(2) Lemma. Let $\mathscr{R}$ be an $S_{\mathscr{E}} P$-class, and let $E=\left\{r_{F} \mid F\right.$ free $\}$ (i.e., the class of reflection maps of free objects). Then, $\mathscr{R} \subseteq$ inj $E \subseteq H \mathscr{R}$.

Proof. $\mathscr{R} \subseteq \operatorname{inj}\{r\}$ for any $\mathscr{R}$-reflection map $r$, whence $\mathscr{R} \subseteq \operatorname{inj} E$.

Let $A \in \operatorname{inj} E$, and (per (1)) let $F$ be free and $F \stackrel{f}{\rightarrow} A$ a surjection. Then, there is $r F \stackrel{\bar{f}}{\rightarrow} A$ with $\bar{f} r_{F}=f$, and $f$ is a surjection $\left(\right.$ since $(\mu \bar{f})\left(\mu r_{F}\right)$ $=\mu f$ is a retraction, whence so is $\mu \bar{f})$. Thus $A \in H \mathscr{R}$.

So, if $\mathscr{R}$ is $H S_{\mathscr{E}} P,(2)$ yields $\mathscr{R}=\operatorname{inj} E$.

We now show that, for $E$ as described, inj $E$ is $H S_{\mathscr{E}} P$. From 1.2, inj $E$ is already $S_{\mathscr{E}} P$, so we show $H$ :

(3) Lemma. (Slight variant of 31.8, [HS].) In $\mathscr{C}$, each free object is "sur-projective": Given $F$ free, $F \stackrel{f}{\rightarrow} Q$ and surjection $A \stackrel{\sigma}{\rightarrow} Q$, there is $F \stackrel{\bar{f}}{\rightarrow} A$ with $\sigma \bar{f}=f$.

Proof. Let $F=F(G)$, and consider

$\begin{array}{ccc}G & \stackrel{\iota_{G}}{\rightarrow} & \mu F(G) \\ \chi^{\prime} \downarrow & \mu \bar{f} \xi^{\prime} & \downarrow \mu f \\ \mu A & \stackrel{\mu \sigma}{\rightleftarrows} & \mu Q \\ & \underset{\chi}{\longrightarrow} & \end{array}$


where, first, $\chi$ exists with $(\mu \sigma) \chi=\mathrm{id}_{u Q}$, since $\sigma$ is a surjection; second, we define $\chi^{\prime}=\chi(\mu f) \iota_{G}$; third, there is unique $F(G) \stackrel{\bar{f}}{\rightarrow} A$ with $(\mu \bar{f}) \iota_{G}=$ $\chi^{\prime}$, by freedom.

By uniqueness of "lifts over $F(G)$ " there is only one $F(G) \stackrel{g}{\rightarrow} Q$ with $(\mu g) \iota_{G}=(\mu \sigma) \chi^{\prime}$. But $\mu(\sigma \bar{f}) \iota_{G}=(\mu \sigma)(\mu \bar{f}) \iota_{G}=(\mu \sigma) \chi^{\prime}$, and also $(\mu f) \iota_{G}$ $=\left(\operatorname{id}_{u Q}\right)(\mu f) \iota_{G}=(\mu \sigma) \chi(\mu f) \iota_{G}=(\mu \sigma) \chi^{\prime}$. Thus $\sigma \bar{f}=f$, as desired.

(4) LemMA. Let $E \subseteq \mathscr{E}$ with domain (e) free, for each $e \in E$. Then, $\operatorname{inj} E$ is an $H$-class.

Proof. Let $A \in \operatorname{inj} E, A \stackrel{\sigma}{\rightarrow} Q$ a surjection, $F \stackrel{e}{\rightarrow} B \in E$ (with $F$ free), and $F \stackrel{f}{\rightarrow} Q$. By (3), there is $F \stackrel{f}{\rightarrow} A$ with $\sigma f=f$. Then there is $B \stackrel{f}{\rightarrow} A$ with $\bar{f} e=\bar{f}$, whence $\sigma(\bar{f} e)=(\sigma \bar{f}) e=\sigma \bar{f}=f$, as desired. So $Q \in \operatorname{inj} E$.

That completes the proof of 2.2 .

2.4. Corollary. Let $\mathscr{C}$ be an $\mathscr{E}$-category with products, which satisfies 2.1, and let $\mathscr{R}$ be an $S_{\mathscr{E}} P$-class. Then: $H \mathscr{R}=\operatorname{inj}\left\{r_{F} \mid F\right.$ free $\}$.

Proof. (2) shows that $H \mathscr{R} \subseteq H \operatorname{inj}\left\{r_{F} \mid F\right.$ free $\} \subseteq H \mathscr{R}$. But $\operatorname{inj}\left\{r_{F} \mid\right.$ $F$ free $\}$ is already an $H$-class, by (4) above.

3. Remark on identities. We derive a version of Birkhoff's Variety Theorem. So the model is the class of abstract algebras of some type. (See [P].) Suppose that $\mathscr{C} \stackrel{\mu}{\rightarrow}$ Sets satisfies 2.1.

A class $E$ of surjections from free objects is called a class of identities; if $A \in \operatorname{inj} E$ we say that $A$ satisfies $E$; inj $E$ is called an equational class. (A single identity may be thought of like this: Let $F$ be free and $p$, $q \in F$. Let $e$ be the quotient of $F$ onto $F$ modulo " $p=q$ ". Then, $A$ satisfies " $p=q$ " means $A \in \operatorname{inj}\{e\}$.)

So let $\mathscr{E}=$ all surjections, suppose that $\mathscr{M}(\mathscr{E})=$ all monics, and suppose $\mathscr{C}$ is an $\mathscr{E}$-category with products. For $S_{\mathscr{E}}$, write $S$. Then 2.2 holds, and reads:

$\mathscr{R}$ is an HSP-class iff $\mathscr{R}$ is an equational class.

4. Applications. For the various specific $\mathscr{C}$ 's below, we shall, unless mentioned otherwise, take $\mathscr{E}=$ all epics, whence $\mathscr{M}(\mathscr{E})$ - the so-called extremal monics consists of monics $A \rightarrow B$ for which, identifying $A$ with 
its image, " $A$ admist no epic enlargement within $B$." For each specific $\mathscr{C}$ below, we shall, of necessity, be quite sketchy about technical details.

4.1. Hausdorff and Tychonoff spaces. We shall recover the theorem of Kannan-Soundararajan [KS]. For Haus, epic means dense image (whence Haus is (epi-) cowellpowered), and extremal subobject means closed subspace. Morphisms then factor as $X \stackrel{f}{\rightarrow} Y=X \rightarrow \overline{f(X)} \hookrightarrow Y$, and Haus is an epi-category, of course with products. Take the usual forgetful Haus $\stackrel{\mu}{\rightarrow}$ Sets; then the free objects $F(S)$ exactly have $\mu F(S)=S$ with $F(S)$ discrete.

So 2.2 applies and says: $\mathscr{R}$ is $H S_{\mathscr{E}} P$ iff $\mathscr{R}=\operatorname{inj} E$, for some class $E$ of dense maps of discrete spaces. About such $\mathscr{R}$, one now proves easily that the following are equivalent. (1) $\mathscr{R}$ contains a space with $\geq 2$ points. (2) $\mathscr{R}$ contains discrete $\{0,1\}$. (3) $\mathscr{R}$ contains every compact space. (4) Each $e \in E$ is compact-extendible (i.e., Comp $\subseteq$ inj $E$ ).

Compact-extendible maps in Haus are described in 2.5.1 of [He], and in [KS] (p. 143, Step 4, (a), ${ }_{e}(\mathrm{~b}),(\mathrm{c})$ ). For simplicity, we restrict to Tychonoff spaces. Here, $D \stackrel{e}{\rightarrow} K$ is compact-extendible iff $K \subseteq \beta D$ (Stone-Čech compactification) and $e$ is an embedding. Rephrasing in Tych: for each discrete $D$, let $E \subseteq K(D) \subseteq \beta D$, let $\mathscr{K}=\{D \hookrightarrow K(D) \mid D$ discrete $\}$, and rename inj $\mathscr{K}$ as “ $\mathscr{K}$-compactness". Now 2.2 for Tych reads: $\mathscr{R}$ is $H S_{\mathscr{E}} P$ iff $\mathscr{R}=\{\{1\}, \varnothing\}$ or $\mathscr{R}=\mathscr{K}$-compactness for some $\mathscr{K}$. [KS] prove this, actually for regular Hausdorff spaces.

(We note that this example can be described with $\mathscr{F}=$ all dense compact-extendible maps, whence $\mathscr{M}(\mathscr{F})=$ all perfect maps. (See $33 \mathrm{H}$ of [HS].) Here, $\mathscr{M}(\mathscr{F}) \varsubsetneqq$ monics. Then, one can show, a class $\mathscr{R}$ is $S_{\mathscr{F}} P$ iff $\mathscr{R}$ is $S_{\mathscr{E}} P$ (i.e., epireflective) and $\mathscr{R}$ contains all compact spaces.)

4.2. Uniform spaces. Unif is the category of Hausdorff uniform spaces. For $X \in$ Unif, $s X$ denotes Samuel compactification. (See $\left[\mathbf{I}_{1}\right]$.) Everything said above about Haus is true in Unif, up to the point of what "compactextendible" means. Here, $D \stackrel{e}{\rightarrow} K$ is compact-extendible iff $s K=s X$ and $e$ is a uniformly continuous homeomorphism (which means that topologically $K$ is a subspace of $s D$, and the uniformity of $K$ is no coarser than the relative one from $s D$, and relativized to $D$, is no finer than $D$ 's- which for discrete $D$ is automatic). So, for each (uniformly) discrete $D$, let $D \stackrel{e}{\rightarrow} K(D)$ be compact-extendible, let $\mathscr{K}=\{D \stackrel{e}{\rightarrow} K(D) \mid D$ discrete $\}$, and rename inj $\mathscr{K}$ as " $\mathscr{K}$-compactness". Then, $\mathscr{R}$ is $H S_{\mathscr{E}} P$ iff $\mathscr{R}=$ $\{\{1\}, \varnothing\}$, or $\mathscr{R}=\mathscr{K}$-compactness for some $\mathscr{K}$. 
4.3. Proximity and Alexandroff spaces. (See $\left[\mathbf{I}_{1}\right]$ and $\left[\mathbf{H}_{1}\right]$, respectively.) Assume the Hausdorff axiom. The $\mathrm{HS}_{\mathscr{E}}$ P-classes in each of these categories are as in Tych, with the Smirnov compactification (Prox) and the Alexandroff compactification (Alex) replacing the Stone-Čech compactification.

4.4. Abelian torsion-free and lattice-ordered groups. [HMa] describes $\mathrm{HS}_{\mathscr{E}} \mathrm{P}$-classes in (let us say) $\mathrm{Ab}$ and $l \mathrm{Ab}$. We sketch the situation.

Here, " $A \stackrel{e}{\rightarrow} B$ is epic" means that $B$ is a subobject of the divisible hull of the image $e(A)$. For $\mathrm{Ab}$, that is elementary and for $l \mathrm{Ab}$, it is a theorem from [AC]. So $A b$ and $l \mathrm{Ab}$ are epi-cowellpowered. Extremal subobject thus means pure subgroup or sub-l-group, and homomorphisms $A \stackrel{h}{\rightarrow} B$ factor as $A \stackrel{h}{\rightarrow} B=A \stackrel{h}{\rightarrow} p(h(B)) \rightarrow B$, where $p(h(B))$ is the pure subobject generated by $h(B)$.

We use the usual underlying-set-functor, $\mathrm{Ab}$ or $l \mathrm{Ab} \stackrel{\mu}{\rightarrow}$ Sets. The free object on the set $S, F(S)$, is for $\mathrm{Ab}, \sum\left\{Z_{s} \mid s \in S\right\}$, (each $Z_{s}=Z$ ), and for $l \mathrm{Ab}$, the sub-l-group of $Z^{Z^{S}}$ generated by all projections $\pi_{s}: Z^{S} \rightarrow Z_{s}$ (see [BKW]). So 2.2 applies, but is reduced further as follows:

(a) $[\mathrm{HMa}]$ In $\mathrm{Ab}$ or $l \mathrm{Ab}$, let $\mathscr{R}$ be $S_{\mathscr{E}} P$. Then, $\operatorname{inj}\left\{r_{F} \mid F\right.$ free $\}=$ $\operatorname{inj}\left\{Z \stackrel{r_{Z}}{\rightarrow} r Z\right\}$.

Then, if $\mathscr{R}$ (still only $S_{\mathscr{E}} P$ ) is not trivial, $r_{Z}$ must be an embedding and it follows from elementary algebra that $r Z$ is a subring of $Q$, hence is the subring of $Q$ generated by reciprocals of some set $P$ of primes, and $A \in \operatorname{inj}\left\{Z \stackrel{r_{Z}}{\rightarrow} r Z\right\}$ means $A$ is $P$-divisible. Thus

(b) [HMa] In $\mathrm{Ab}$ or $l \mathrm{Ab}, \mathscr{R}$ is $\mathrm{HS}_{\mathscr{E}} \mathrm{P}$ iff $\mathscr{R}=\{(0)\}$ or $\mathscr{R}=P$-divisible, for some set $P$ of primes.

4.5. Archimedean l-groups with strong unit. The details appear in [HMo]. This category generalizes Banach lattices with unit, a topic of interest in functional analysis.

We admit the case of unit $=0$; i.e., the zero-group is an object. Morphisms are $l$-group homomorphisms preserving unit. For simplicity in describing epics, etc., we shall assume here that all groups are divisible. Let $\mathscr{S}$ be the category. We require the Yosida representation: Each non-zero $A \in \mathscr{S}$ is isomorphic to $\hat{A} \subseteq C\left(Y_{A}\right), Y_{A}$ compact Hausdorff, with $\hat{u}_{A}=1\left(u_{A}\right.$ the unit of $\left.A\right)$ and $\hat{A}$ separating points of $Y_{A}$. We identify $A$ with $\hat{A}$.

Then: $A \stackrel{h}{\rightarrow} B$ is epic iff $h(A)$ is uniformly dense in $B$; whence $\mathscr{S}$ is epi-cowellpowered. And for a subobject, "extremal" means uniformly 
closed. So morphisms factor as $A \stackrel{h}{\rightarrow} B=A \rightarrow \overline{h(A)} \hookrightarrow B$, “( $)$ " denoting uniform closure.

$\mathscr{S}$ has products: Given $\left\{A_{i}\right\}$ with units $\left\{u_{i}\right\}$, the $\mathscr{S}$-product is the ideal in the l-group product ("cardinal product") generated by $u=\left\langle u_{i}\right\rangle$, with $u$ as unit.

Take $\mathscr{S}^{\mu} \rightarrow \mathscr{G}$ as: The objects of $\mathscr{G}$ are partially ordered sets with top and bottom (possibly equal), and no other order relations, with maps preserving top and bottom as morphisms. $\mu(A) \equiv\left\{a \in A|| a \mid \leq u_{A}\right\}$ (the unit ball), with the $\mathscr{G}$-order ( $u_{A}=$ top, $-u_{A}=$ bottom).

Then: $A \stackrel{\sigma}{\rightarrow} B$ is a "surjection" iff either $A=B=(0)$, or, $B \neq(0)$ and $\sigma$ is an onto homomorphism.

An object of $\mathscr{G}$ is either a singleton, whence the associated free object is (0), or it can be unambiguously expressed in the form $G \cup\{ \pm 1\}$. Then, the free object over $G \cup\{ \pm 1\}$, say $F(G)$, is the l-subgroup of $C\left([-1,1]^{G}\right)$ generated by 1 and all projections $\pi_{g}:[-1,1]^{G} \rightarrow[-1,1]$. (Thus $F(\varnothing)$ is the reals.)

2.2 applies, and is reduced further as follows:

(a) If $\mathscr{R}$ is $S_{\mathscr{E}} P$, then $\operatorname{inj}\left\{r_{F} \mid F\right.$ free $\}=\operatorname{inj}\{F(\omega) \rightarrow r F(\omega)\}$.

Then, if $\mathscr{R}$ (still only $S_{\mathscr{E}} P$ ) is not trivial, $F(\omega) \rightarrow r F(\omega)$ must be an embedding, and $r F(\omega) \subseteq C\left([-1,1]^{\omega}\right)$ follows.

When $\mathcal{O} \subseteq C\left([-1,1]^{\omega}\right)$, define the class " $\mathcal{O}$-closed" as: $A$ is $\mathcal{O}$-closed $\equiv$ whenever $a_{1}, a_{2}, \ldots, \in A$ with $\left|a_{i}\right| \leq 1$, and $0 \in \mathcal{O}$, then $0 \circ\left\langle a_{i}\right\rangle \in A$. (For each $i$, we have $a_{i}: Y_{A} \rightarrow[-1,1]$, whence we have the "evaluation map" $\left\langle a_{i}\right\rangle: Y_{A} \rightarrow[-1,1]^{\omega}$. Thus $0 \circ\left\langle a_{i}\right\rangle: Y_{A} \rightarrow R$, and $0 \circ\left\langle a_{i}\right\rangle$ may or may not be an element of $A$.)

Let [O $\mathcal{O}$ be the $l$-subgroup generated by $F(\omega) \cup \mathcal{O}$.

(b) $A$ is $\mathcal{O}$-closed iff $A \in \operatorname{inj}\{F(\omega) \hookrightarrow[\mathcal{O}]\}$.

(c) $\mathscr{R}$ is $\mathrm{HS}_{\mathscr{E}} \mathrm{P}$ iff $\mathscr{R}=\{(0)\}$ or $\mathscr{R}=\mathcal{O}$-closed, for some $\mathcal{O}$.

(The referee points out that the above example can be described just as well using the functor $\mathscr{S} \stackrel{\nu}{\rightarrow}$ Sets with $\nu(A) \equiv$ the open unit ball of $A$ : here, $\nu=\alpha \mu$, where $\mathscr{G} \stackrel{\alpha}{\rightarrow}$ Sets removes top and bottom; then $\alpha$ has left adjoint "adjoint top and bottom", and the object of $\mathscr{S}$ is $\mu$-free iff it is $\nu$-free.)

4.6. Archimedean l-groups with weak unit. This situation is detailed in $\left[\mathbf{H}_{2,3}\right]$. Here we take $\mathscr{E}=$ all essential epics, $\mathscr{G}=$ pointed sets, and $\mu(A)$ is the underlying set of $A$ with the weak unit as base point. Then, the description of the $\mathrm{HS}_{\mathscr{E}} \mathrm{P}$-classes is similar to $4.5(\mathrm{c})$, with $C\left(R^{\omega}\right)$ replacing $C\left([-1,1]^{\omega}\right)$. (Also, one can use a functor $\nu$ directly to Sets, as at the end of 4.5.) 
The analysis of this situation is not yet complete. I don't know whether or not the above describes all epireflective $H$-closed classes. Only recently have the epics been characterized, by $\mathrm{R}$. N. Ball and the present author, and we don't know yet what the extremal monics are.

5. Remark on implicit operations. Suppose in $\mathscr{C}$ that 2.2 holds for $\mathscr{E}=$ all epics. All of the examples in $\S 4$ except Unif have the property that an $\mathrm{HS}_{\mathscr{E}} \mathrm{P}$-class inj $E$ is nontrivial iff each $e$ is an embedding. It seems reasonable to call a class $E$ of epic embeddings of free objects a class of operations, and, if $A \in$ inj $E$, to say that $A$ is $E$-closed. Roughly speaking, given the epic embedding $F \rightarrow B$ of free $F$, the elements of $F$ are "explicit operations" and the elements of $B-F$ are "implicit". If $F$ is free on a set of power $m$, then these operations are thought of as having arity $m .4 .4$ and 4.5 especially support that view, and with that view, 4.4 says that in $\mathrm{Ab}$ or $l \mathrm{Ab}$, every implicit operation is unary, and 4.5 says that in $\mathscr{S}$, every implicit operation is $\leq \omega$-ary. In the same vein, one sees that in Tych there is no cardinal bound on arity of implicit operations.

There seems to be a cardinal property of (say, for simplicity) $\mathscr{C} \stackrel{\mu}{\rightarrow}$ Sets with enough free objects, namely "maximal true arity of implicit operations" defined perhaps as:

$\alpha \equiv$ least $m$ such that for every class $E$ of operations, there is a class $E^{\prime}$ of $\leq m$-ary operations (i.e., the domains are free on sets of power $\leq m$ ) such that $A$ is $E$-closed iff $A$ is $E^{\prime}$-closed (i.e., inj $E=\operatorname{inj} E^{\prime}$ ).

Usually then, for such $E^{\prime}$, there will be a single morphism $e$ such that $\operatorname{inj} E^{\prime}=\operatorname{inj}\{e\}$ - as in 4.4 and 4.5 .

Acknowledgment. Thanks are due the referee and F. E. J. Linton for several observations and suggestions.

\section{REFERENCES}

[AC] M. Anderson and P. Conrad, Epicomplete l-groups, Alg. Univ., 12 (1981), 224-241.

[BKW] A. Bigard, K. Keimel, and S. Wolfenstein, Groupes et Anneaux Reticules, Springer Lecture Notes 608, Berlin, 1977.

$\left[\mathrm{H}_{1}\right] \quad$ A. W. Hager, Cozero fields, Conf. Sem. Di. Math. Univ. Bari, 175 (1980).

$\left[\mathrm{H}_{2}\right] \quad, \quad C(X)$ has no proper functorial hulls, Proc. Special Session on Rings of Continuous Functions, Cincinnati 1982, Marcel Dekker, to appear.

$\left[\mathrm{H}_{3}\right] \quad$ Algebraic closures of l-groups of continuous functions, ibid.

[HMa] A. W. Hager and J. J. Madden, Algebraic classes of abelian torsion-free and lattice-ordered groups, to appear.

[HMo] A. W. Hager and C. M. Monaco, On the category of archimedean l-groups with strong unit, in preparation. 
[He] H. Herrlich, Topologische Reflexionen and Coreflexionen, Springer Lecture Notes 78, Berlin 1966.

[HS] H. Herrlich and G. Strecker, Category Theory, Allyn and Bacon, Boston, 1973.

[I $\left.\mathrm{I}_{1}\right] \quad$ J. R. Isbell, Uniform spaces, Amer. Math. Soc., Providence, 196.

[ $\left.\mathrm{I}_{2}\right]$ _ Natural sums and abelianizing, Pacific J. Math., 14 (1964), 1265-1281.

$\left[\mathrm{I}_{3}\right] \quad$, Structure of categories, Bull. Amer. Math. Soc., 72 (1966), 619-655.

$\left[\mathrm{K}_{1}\right] \quad$ J. F. Kennison, A note on reflection maps, Illinois J. Math., 11 (1967), 404-409.

$\left[\mathrm{K}_{2}\right] \quad$, Full reflective subcategories and generalized covering spaces, Illinois $\mathrm{J}$. Math., 12 (1968), 353-365.

[KS] V. Kannan and T. Soundararajan, Properties that are productive, closed-hereditary and surjective, Topology and its Appl., 12 (1981), 141-146.

[P] R. S. Pierce, Introduction to the Theory of Abstract Algebras. Holt, Rhinehart, and Winston, NY (1968).

Received July 9, 1984 and in revised form January 10, 1985.

WESLEYAN UNIVERSITY

MidDLETOWN, CT 06547 


\section{PACIFIC JOURNAL OF MATHEMATICS EDITORS}

\author{
V. S. VARADARAJAN \\ (Managing Editor) \\ University of California \\ Los Angeles, CA 90024 \\ HERBERT ClEMENS \\ University of Utah \\ Salt Lake City, UT 84112 \\ R. FINN \\ Stanford University \\ Stanford, CA 94305
}

\author{
HERMANN FLASCHKA \\ University of Arizona \\ Tucson, AZ 85721 \\ RAMESH A. GANGOLLI \\ University of Washington \\ Seattle, WA 98195 \\ VAUghan F. R. JONES \\ University of California \\ Berkeley, CA 94720 \\ ROBION KIRBY \\ University of California \\ Berkeley, CA 94720
}

C. C. MOORE

University of California Berkeley, CA 94720

H. SAMELSON

Stanford University Stanford, CA 94305

HAROLD STARK

University of California, San Diego La Jolla, CA 92093

\section{ASSOCIATE EDITORS}
R. ARENS
E. F. BECKENBACH
B. H. NEUMANN
F. WOLF
K. YOSHIDA (1906-1982)

\section{SUPPORTING INSTITUTIONS}

UNIVERSITY OF ARIZONA

UNIVERSITY OF BRITISH COLUMBIA

CALIFORNIA INSTITUTE OF TECHNOLOGY

UNIVERSITY OF CALIFORNIA

MONTANA STATE UNIVERSITY

UNIVERSITY OF NEVADA, RENO

NEW MEXICO STATE UNIVERSITY

OREGON STATE UNIVERSITY
UNIVERSITY OF OREGON UNIVERSITY OF SOUTHERN CALIFORNIA

STANFORD UNIVERSITY

UNIVERSITY OF HAWAII

UNIVERSITY OF TOKYO

UNIVERSITY OF UTAH

WASHINGTON STATE UNIVERSITY

UNIVERSITY OF WASHINGTON 


\section{Pacific Journal of Mathematics}

Vol. 125, No. 1 September, 1986

Gilles Christol, Fonctions et éléments algébriques $\ldots \ldots \ldots \ldots \ldots \ldots \ldots \ldots$

Jo-Ann Deborah Cohen, Extensions of valuation and absolute valued

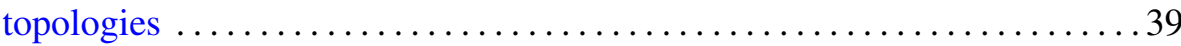

Miriam Cohen, Smash products, inner actions and quotient rings . . . . . . 45

Mikio Furushima, On the singular $K-3$ surfaces with hypersurface

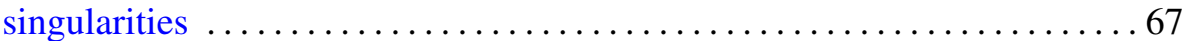

Gerhard Gierz and Boris Shekhtman, A duality principle for rational

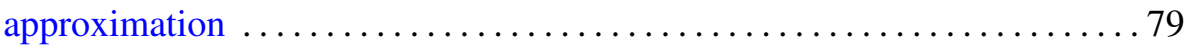

Anthony Wood Hager, A description of HSP-like classes, and

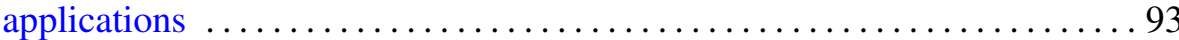

George Alan Jennings, Lines having high contact with a projective

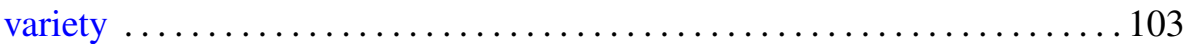

John Lott, Eigenvalue bounds for the Dirac operator . . . . . . . . . . . 117

Denis Laurent Luminet, A functional calculus for Banach PI-algebras . . . . 127

Shizuo Miyajima and Noboru Okazawa, Generators of positive

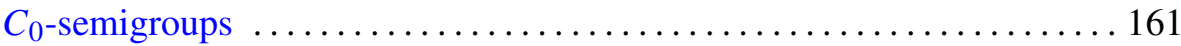

Takemi Mizokami, On functions and stratifiable $\mu$-spaces $\ldots \ldots \ldots \ldots \ldots 177$

Jeff Parker, 4-dimensional $G$-manifolds with 3-dimensional orbits . . . . . 187

Elias Saab and Paulette Saab, On Peł czyński's properties (V) and (V*) . . 205

Elmar Schrohe, The symbols of an algebra of pseudodifferential operators

Aart van Harten and Els Vader-Burger, Approximate Green functions as a tool to prove correctness of a formal approximation in a model of competing and diffusing species

Stephen Watson, Using prediction principles to construct ordered

continua 